\title{
Magnetic Activity at Very Low Masses
}

\author{
Gibor Basri \\ Astronomy Department, MC3411, \\ University of California, Berkeley, 94720, USA
}

\begin{abstract}
The characteristics of stars of very low mass are reviewed with emphasis on signatures of and consequences of magnetic activity.
\end{abstract}

\section{Introduction}

One of the clearest violations of the Russell-Vogt theorem (which holds that mass sets everything in stars) is the level of magnetic activity in stellar atmospheres. It is now well established that for solar-type stars, the activity is determined primarily by the angular momentum of the star (which in turn is diminished by the magnetic wind). Production of the cyclical field on the Sun is through a dynamo operating at the bottom of the convection zone. It is thought to be of the $\alpha \omega$ variety, operating in the shear layer between the convective and radiative zones. As the convection zone deepens, either in stars of smaller mass or at the end of the main sequence phase, such a dynamo must eventually fail. The solar field at activity minimum is dominated by a pervasive smaller-scale dynamo, which may be distributed throughout the convection zone. In fully convective pre-main sequence stars, or stars with masses less than $0.3 \mathrm{M}_{\odot}$, there is no shear boundary. Dynamos in these stars must either be turbulent (which would only weakly depend on rotation), or of the $\alpha^{2}$ variety (which would have a stronger rotational dependence). A good review of this overall subject can be found in Charbonneau et al. (1997).

The nature of the $\alpha \omega$ dynamo naturally leads to a connection between angular momentum and total magnetic flux. A connection between the surface rotation of a convective star and the amount of magnetic heating in the atmosphere has been noted since the 1960s (eg. Kraft 1967). Magnetic activity is diagnosed by CaII emission, $\mathrm{H} \alpha$ emission, UV emission lines (from the IUE satellite onward) or coronal (X-ray) emission (from the Einstein satellite onward). The activity can be characterized by the surface flux in the given diagnostic; this is to be preferred over a simple luminosity as it accounts for the variations in stellar radii.

More commonly it is expressed as a luminosity ratio between the diagnostic and the stellar bolometric luminosity (not requiring knowledge of the stellar parameters beyond a bolometric correction). Use of this ratio assumes that there is some physical connection between the two luminosities. This receives some empirical support in the behavior of the ratio, although it should be pointed out that there are a great many steps between the operation of a dynamo at the bottom of the convection zone and the production of hot plasma at the top 
of the atmosphere. Any one of these could serve as a valve which controls the tiny tap magnetic activity represents on the stellar bolometric luminosity, so it is perhaps surprising that the ratio works as well as it does.

Stellar rotation is relatively easy to measure. It is usually measured through Doppler broadening of spectral lines (which only gives the projected rotation velocity), or by detecting a periodic rotational modulation of magnetic features (usually utilizing a broadband luminosity). Noyes et al. (1981) showed that for solar-type stars, the activity characterized by luminosity ratios is a strong function of the Rossby number (the ratio of the rotation period to a convective eddy timescale, properly chosen). A large literature has since accumulated confirming the rotation-activity connection in solar-type stars. Basri (1986) pointed out that there is also a good (and perhaps even equivalent) rotation-activity connection between the surface flux of any activity diagnostic (since they are all well correlated with each other) and rotation period. This is because the Rossby number is related to the bolometric luminosity through the convective overturn times. Pizzolato et al. (2003) have recently rediscovered this point (see also Montesinos et al. 2001).

There is no reason why only one dynamo mode must be active. The Sun at minimum activity may be displaying primarily a turbulent mode, while the cyclic increase and decrease (and extra activity therein) could be due to the $\alpha \omega$ dynamo. The relative contributions of these two probably shifts as stars have deeper convection zones, with the importance of the $\alpha \omega$ dynamo diminishing in cooler stars. The $\alpha \omega$ dynamo is also responsible for the large loop structures on the Sun (as it arises from deep layers). It has been argued that the behavior of angular momentum in early $\mathrm{M}$ stars can be explained in part by the configuration of the field changing to smaller (less open) loops, which in turn would reduce the amount of magnetic braking. As the $\alpha \omega$ dynamo is being quenched by the disappearance of the radiative zone, the magnetic fields on the star will be switching over to full production from turbulent or $\alpha^{2}$ dynamos. Near the fully convective boundary (at about spectral type M3 for dwarfs), the fields would already be coming almost entirely from the latter types of dynamo. This would explain why nothing special seems to happen at the fully convective boundary for main sequence stars.

A further phenomenon is found in rotation-activity studies, called "saturation". This is a tendency for the amount of activity to achieve a maximum at a certain rotation, beyond which increasing the rotation no longer increases the activity. The reasons for this are not understood; it may be a characteristic of the dynamo production of fields, it may be connected to the heating mechanisms and their connection to density or emission measure, or it may reflect full coverage of the surface or atmospheric volume by fields (with no room to add more). It is known that more active stars are that way primarily because of increased filling factors rather than field strengths (Saar 1996). Another possibility is that saturation is due to centrifugal stripping of the corona (Jardine \& Unhuh 1999).

The activity levels on the Sun are well below saturation even at its most active. $\mathrm{L}_{X} / \mathrm{L}_{b o l}$ for the Sun is in the range of $10^{-6}$, while this quantity saturates at about $10^{-3}$ for very active $M$ stars (Pizzolato et al. 2003). The rotation velocity needed to cause saturation is about $35 \mathrm{~km} \mathrm{~s}^{-1}$ for early $\mathrm{G}$ stars, 10 $\mathrm{km} \mathrm{s}^{-1}$ for $\mathrm{K}$ stars, dropping to $5 \mathrm{~km} \mathrm{~s}^{-1}$ for mid-M stars (Mohanty \& Basri 
2003, Pizzolato et al. 2003). In terms of rotation periods, these are about 1.5 days for $\mathrm{G}$ stars, 4 days for $\mathrm{K}$ stars, and 3 days for $\mathrm{M}$ stars. There may also be a phenomenon called "super-saturation", in which extremely rapidly rotating stars are less active than the saturation limit (eg. James et al. 2000). For M stars the rotation-activity relation below saturation is not well known, because unsaturated stars are generally rotating too slowly to obtain a specific value for rotation velocity, and too few slow rotators have been found cooler than M4.

\section{Rotation at the Lowest Masses}

Very little was known about stars later than M5 before the era of $8-10 \mathrm{~m}$ class telescopes which began in the mid 1990s. The spaceborne observatories prior to Chandra and XMM-Newton also had too little sensitivity to the much smaller fluxes of high energy radiation from these objects (even if they have large surface fluxes, they have small surfaces). The study of the rotation-activity relation in such objects began on the Keck I telescope using the HIRES echelle (Basri \& Marcy 1995). One of the coolest known objects at the time, BRI 0021-0214 (M9.5), was found to have a bafflingly smooth spectrum, even at high resolution. A cross-correlation analysis with slowly rotating templates showed great Doppler smearing from rotation at 20 times the usual rate for $M$ field dwarfs (less than a 3 -hour rotation period!). Normally such rapid rotation would certainly be accompanied by saturated stellar activity, but there was no (with an upper limit well under $1 \AA$ ) observed $\mathrm{H} \alpha$ emission (henceforth we will use $\mathrm{H} \alpha$ as a proxy for magnetic activity unless otherwise stated).

Over the next few years, many more cool objects were observed for rotation and activity : Basri (1996), Tinney \& Reid (1998), Delfosse et al. (1998), Basri (2000), Reid et al. (2002), Mohanty \& Basri (2003). Confirmation of the very short rotation periods were also found via photometric monitoring, which detected low amplitude periodic modulation in some very cool objects: Martín, Zapatero-Osorio \& Rebolo (1996), Terndrup et al. (1999), Clarke, Tinney \& Covey (2002), Gelino et al. (2002). I have summarized the rotational results at greater length in Basri (2003). The spindown times of early M stars are somewhat longer than $\mathrm{G}$ or $\mathrm{K}$ dwarfs, but most of the field stars are slow rotators. As one moves into mid-M dwarfs, the incidence of more rapid (5-10 km/s) rotators increases. Spindown times appear to be longer, but slow rotators are still found. Cooler than about M8, it becomes difficult to find slow rotators, and the maximum rotations increase to several tens of $\mathrm{km} / \mathrm{s}$. In the $\mathrm{L}$ dwarfs, th is trend continues with a complete absence of slow rotators, and periods as short as a couple of hours.

Recent results on the rotations of very young, very low mass objects (White \& Basri 2003, Jayawardhana et al. 2002, Basri 2003) suggest that they begin, like their higher mass $\mathrm{T}$ Tauri analogs, with modest rotations (perhaps due to disk locking). After deuterium burning is over, they begin to contract and spin up. If low enough in mass, they begin in the M6-M8 range, and cool to later types after deuterium burning. Magnetic braking apparently becomes ineffective or absent in these cooler types, so they retain the short periods they acquire during contraction. 


\section{Activity at the Lowest Masses}

\section{1. $\quad \mathbf{H} \alpha$ Emission}

Basri \& Marcy (1995) noted that as one goes to late M stars, the equivalent width of $\mathrm{H} \alpha$ becomes increasingly sensitive to chromospheric heating. In order to see $\mathrm{H} \alpha$ at all, one must have non-radiative heating, since the photospheric temperatures do not allow for any substantial population of hydrogen in levels above the ground state. Furthermore, $\mathrm{H} \alpha$ is seen against the continuum at $656 \mathrm{~nm}$, which is in the Wein tail of the spectral energy distribution for late $\mathrm{M}$ objects. Thus, a given surface flux will correspond to a rapidly growing equivalent width (the "contrast effect"). The observed saturated surface fluxes near M5 should yield equivalent widths of more than $100 \AA$ by M9.

In reality, $\mathrm{H} \alpha$ equivalent widths seem to peak at $10-15 \AA$, regardless of spectral type or age (eg. Stauffer et al. 1995; Gizis et al 2000). Since X-rays remain saturated to late spectral types (Fleming et al. 1993), this cap on $\mathrm{H} \alpha$ strengths is truly puzzling. The source function for $\mathrm{H} \alpha$ is not even well-coupled to local temperature (NLTE line formation) so the level of emission need not be directly related to the amount of non-radiative heating. The continuum is formed in a different (lower) part of the atmosphere. A mechanism for keeping the maximum $\mathrm{H} \alpha$ equivalent widths relatively constant for all late $\mathrm{M}$ stars has not been thought of.

In the case of BRI 0021, one might have posited that the lack of emission was due to the star being a very slow rotator, but quite the opposite is the case. The observed properties of BRI 0021 are therefore remarkable indeed. I re-observed the object several times, without finding emission. However, an $\mathrm{H} \alpha$ flare was later observed on the object (Reid et al. 1999). Since the initial discovery, many more cool objects were observed for rotation and activity: Basri (1996), Tinney \& Reid (1998), Delfosse et al. (1998), Basri (2000), Reid et al. (2002), Mohanty \& Basri (2003), Pizzolato et al. (2003). Much of what was guessed at by Basri $\&$ Marcy (1995) turns out to be the case. The $\mathrm{H} \alpha$ surface flux begins a decline at M6 or M7, and the decline becomes precipitous at around M9. Along with the rotational results described above, there is clear evidence suggesting that the magnetic brakes are being turned off.

Large surveys of $\mathrm{H} \alpha$ emission in clusters have been carried out by Stauffer and co-workers (eg. Stauffer et al. 1997; Barrado et al. 1998). The largest compendia of $\mathrm{H} \alpha$ emission for field stars have come from the Palomar M-star survey (Gizis, Reid, \& Hawley 2002). Further work on L stars has been published by Gizis et al. (2000) and in this volume. These show that the fraction of stars showing emission increases from early $M$ subclasses into mid M subclasses. This is due primarily to the contrast effect discussed above. Although Gizis et al. (2000) found that the fraction of stars with emission approached $100 \%$ at M5, their later results have reduced this somewhat (see references by Hawley and co-workers in this volume). They continue to find, however, that the fraction decreases again at late spectral subclasses. This cannot be due to the contrast effect (which only gains strength with cooler temperatures). The situation becomes even clearer for L dwarfs. As noted by Basri and Gizis and co-workers in Cool Stars 11 (2000), the fraction of $\mathrm{L}$ dwarfs showing $\mathrm{H} \alpha$ emission at all is rather low, and those which do tend to have very weak lines (or are flaring). 
Weak emission in an L dwarf corresponds to a very low surface flux; and by mid L subclasses there are essentially no objects left with any emission.

The early L subclasses contain both very low mass stars and brown dwarfs, but the later L subclasses are entirely substellar. It is not clear whether being substellar should matter for chromospheric heating. Both types of objects are usually considered fully convective (see Mullan \& McDonald 2001 for a possible objection to that), although there certainly is a difference in their interiors. The stellar objects have nuclear burning cores (with some continuing gravitational contraction for almost all objects M8 or later, as the pre-main sequence phase for such objects is essentially the lifetime of the Galaxy). In practice, many of the field brown dwarfs found so far (because they are brightest) are probably over 60 jupiter masses and not much over 1 Gyr old (for such objects their substellar status is not obvious). These will still have some nuclear burning at their center, so being technically substellar seems unimportant. Field objects M8 or later showing lithium, however, do not have nuclear burning cores (Basri 1997). Such brown dwarfs have degenerate cores and only distributed heating by gravitational contraction. It is not known how this would impact dynamo activity (if at all). In this category is included the brown dwarf LP 944-20, which shows both continuous (but weak) $\mathrm{H} \alpha$ emission and flaring activity. The drop in activity at M9, therefore, is not due to interior changes, or a switch to brown dwarfs.

\subsection{The Death of Stellar Activity}

In the field, Hawley and Johns-Krull (2003) have shown that there is hot plasma present even in objects with low $\mathrm{H} \alpha$ surface fluxes, in approximate line with expectations from scaling correlations between the various diagnostics. Objects like LP 944-20, which have weak $\mathrm{H} \alpha$ lines, likely have weak coronae as well. Chandra and XMM-Newton observations of this object (Rutledge et al 2000; Martín \& Bouy 2003) placed upper limits on the quiescent corona for this object at $\mathrm{L}_{X} / \mathrm{L}_{b o l}$ at most the level of the quiet Sun. This is three orders of magnitude below the saturation limit, even though the object is a rapid rotator.

An early suggestion for the drop in activity was made by Basri \& Marcy (1995). They pointed out that the convective velocities are becoming rather low for extremely low-luminosity objects (since there is not much energy which must be carried out). The convective overturn times are getting long, but the observations show that the rotation periods are getting quite short. This puts the objects in a regime of very small Rossby numbers, and it is possible that the dynamo action is affected by this (perhaps analogous to super-saturation for rapidly spinning $\mathrm{W}$ Uma stars and others as mentioned above). Mohanty \& Basri (2003) find, however, that Rossby number does not serve to sort out the varying activity levels at the bottom of the main sequence.

A more promising explanation was first advanced by Meyer \& MeyerHofmeister (1999). They were studying the period gap in cataclysmic variables, and suggested that it is due to the fact that very low mass companions might not provide magnetic rotational braking due to their very neutral atmospheres. The idea is that if the atmosphere (which, after all, is all we can see) is sufficiently neutral, the field freezing which normally occurs in outer stellar atmospheres will cease to operate. The atmospheric motions due to convection will cease to 
force the field into non-potential configurations, or propagate magneto-acoustic waves upwards. These two mechanisms are thought to lie at the heart of the non-radiative heating mechanisms that produce stellar activity. The acoustic (basal) flux should also have fallen very low, because of the decreased convective velocities (acoustic luminosity varies with the eighth power of velocity).

The problem boils down to evaluating a magnetic Reynolds number. A stellar atmosphere, of course, has a run of temperatures and pressures, so it is better to use real atmospheres (with a proper evaluation of convective velocities) to check whether atmospheric motions can generate a perturbed field, given some background field which rises from the hotter (coupled) deep atmosphere. Meyer \& Meyer-Hofmeister (1999) used an approximate treatment, and found that atmospheric neutrality is a plausible explanation for a lack of activity. Mohanty et al. (2002) performed a more detailed analysis, using atmospheres for $1500-3000 \mathrm{~K}$ from the Lyon group. They examined a variety of effective temperatures, and used convective and turbulent velocities to see whether a reasonable perturbed field could be generated.

They found that the fractional ionizations in these atmospheres are very low, ranging from $10^{-6}-10^{-7}$ at the photospheres to orders of magnitude less in the upper atmospheres. The magnetic Reynolds number provides an estimate of how much additional field will be produced if one moves the atmosphere through the field (generating currents). If there are few charged particles, not much current is generated, and if the density of neutrals is high enough, even the charged particles cannot keep an association with a given field line (they are pushed off by neutral collisions). The very low ionization fractions in these atmospheres lead to Reynolds numbers of at most 0.1 at all points in all atmospheres at all levels, given convective velocities. One does not expect much activity to be generated unless the Reynolds numbers are greater than unity. If one assumes velocities 100 times greater (which is still subsonic), the hotter atmospheres attain Reynolds numbers greater than unity in the photosphere for effective temperatures above about $2700 \mathrm{~K}$. At sonic velocities, the temperature at which reasonable nonpotential fields might be generated corresponds loosely to spectral type M9. Thus, neutrality shows promise as an explanation of the death of stellar activity at the bottom of the main sequence.

A question arises when one examines activity in very young objects compared with field objects. X-rays have been detected from a number of brown dwarfs in star-forming regions (Neuhauser et al. 1999). These are typically in the spectral range M6-M8, so it is not completely surprising that they should have activity. The coronal luminosities, however, are larger than for similar spectral types in the field. Part of this is undoubtedly the fact that the brown dwarfs are larger when they are very young. This puts them within the sensitivity range of current X-ray observatories, while it has been much harder to detect coronae on older brown dwarfs. This effect must be carefully controlled for.

Recent studies of M8-M9 objects in star-forming regions (White \& Basri 2003, Jayawardana et al. 2002, and unpublished further work) generally find $\mathrm{H} \alpha$ strengths that are at least several angstroms, rather than the few angstroms that are seen in field dwarfs (if they show anything at all). This is after controlling for the effect of accretion on $\mathrm{H} \alpha$; those authors demonstrate that can 
be ascertained by measuring the breadth of the emission feature. There is even some indication that $\mathrm{L}$ and $\mathrm{T}$ dwarfs might also show $\mathrm{H} \alpha$ emission at very young ages (Zapatero-Osorio et al. 2002a,b). It is also clear that some of the young very late-type objects have really low masses, quite possibly extending into the planetary mass domain (Mohanty et al 2004). It will be fruitful to try to carefully determine whether there is a general enhancement at fixed temperature (spectral type is more problematic) of activity for very young and cool objects. Two possibilities for such an enhancement are 1) some effect of primordial fields still present in the forming objects, and 2) that because they have smaller gravities the ionization fractions will be a little larger.

\subsection{The Flaring Mystery}

M stars are famous for having a (younger) subset which shows large flares (the flare stars). These apparently occur on stellar scales, and can have luminosities which temporarily approach that of the star. This scale is a bit contradictory to the general thought that turbulent dynamos should produce many small loops. At very low masses, the drop in activity might be expected to be accompanied by a drop in flaring. The attempt to detect a quiescent corona on LP 944-20 with Chandra yielded an unexpected bonus (Rutledge et al. 2002). An X-ray flare (with the power of a small solar flare) was seen near the end of the observing period. This result was not entirely surprising, given the flares that had been seen on VB10 (Linsky et al. 1995) and BRI 0021 (Reid et al. 1999), but it was certainly serendipitous. It lent credibility to searching for flares on brown dwarfs, which was quickly rewarded at the VLA. Berger et al. (2002) found fairly frequent flares on this object, and detected quite a number of flares on other objects. These flares are unexpectedly bright in the radio - up to $10^{4}$ times as bright as might be expected from the usual relation between X-ray and radio flare luminosities! They are seen on objects with very little $\mathrm{H} \alpha$ activity, extending well into the $\mathrm{L}$ dwarf domain. Apparently the flare mechanism is rather different than the usual solar analogy (or even the flare star analogy).

This presents a puzzle. The same diminution that is seen in activity might be expected for flare frequencies and luminosities. It is hard to see how one can get a flare without twisting magnetic fields which control substantial plasma into a reconnection configuration. While the breakdown of the normal relation between radio and X-ray luminosity implies a different flare mechanism, it does not relieve the requirement of a non-potential field. Mohanty et al. (2002) looked at the possibility of something analogous to lightning providing the current channel. One only has to go $50-100 \mathrm{~km}$ below the photosphere before the ionization fraction recovers enough to allow good coupling of the plasma to convective motions. Reconnection can occur there; the problem is to get the process to heat its way to the surface where it can be seen. Unfortunately, the current dissipation is very strong along the way (and the timescale for re-neutralization pretty short in this high density medium), so this mechanism is not obviously successful.

Here I present another cartoon idea that is perhaps worth a look. By the very presence of flares we know that there are reasonable fields on these stars above the surface. We also know that not too far below the surface things the field will couple to the plasma. Suppose one has emerging flux regions 
with various polarities (as on solar-type stars). A potential loop sitting above the surface could be impinged on by another rising loop with an incompatible configuration. If there was enough plasma up there, reconnection could begin. As it gets underway, it would accelerate particles, which causes further heating, and creates more plasma. Plasma would boil up the loops in the usual way if the flare really got under way, and further enhance field dissipation.

The main problem, therefore, is to have enough plasma high in the loops to initiate the process. It may be that any object which shows any quiescent $\mathrm{H} \alpha$ emission (like LP 944-20) already has enough hot plasma to get started. The problem is most acute for objects like BRI 0021, which appear not to have any quiescent chromosphere. High in the atmosphere, the resistivities are naturally lower just due to the lower densities. The ionization fraction may also be a bit enhanced by the interstellar UV field. Finally, many of these objects (like BRI 0021) are rotating rapidly enough to substantially reduce the effective gravity in extended loops (cf. Jardine \& Unhuh 1999). Perhaps some combination of these factors allows a relatively low density flare to begin, and it can bootstrap itself. The low X-ray vs radio luminosity may indicate that the densities remain lower than normal, and there is less thermal heating of denser plasma. At the moment, this is nothing more than another hypothesis to be developed.

\section{References}

Barrado y Navascus, D., Stauffer, J.R., \& Randich, S. 1998, ApJ, 506, 347

Basri, G. 1986, Fourth Cambridge Workshop on Cool Stars, Stellar Systems, and the Sun, M. Zeilik \& D. M. Gibson (eds.), Lecture Notes in Physics, (Springer-Verlag : New York), v. 284, p. 184

Basri, G. \& Marcy, G.W. 1995, AJ, 109, 762

Basri, G., Oppenheimer, B.R., Kulkarni, S., Nakajima, T. \& Marcy, G.W. 1996, Ninth Cambridge Workshop on Cool Stars, Stellar Systems, and the Sun, A.S.P. Conf. Ser. 109, R. Pallavicini \& A. K. Dupree, (eds.), 587

Basri, G. 1997, Brown Dwarfs and Extrasolar Planets, A.S.P. CS-134, R. Rebolo, E. Martn, M. R. Zapatero-Osorio eds.), 394

Basri, G. 2000, Eleventh Cambridge Workshop on Cool Stars, Stellar Systems, and the Sun, A.S.P. CS-223, R. Garca-Lpez, R. Rebolo, M. R. ZapateroOsorio (eds.), 261

Basri, G. 2003, IAU Symposium 215, in press.

Berger, E. 2002, ApJ, 572, 503

Charbonneau, P., Schrijver, C.J., \& McGregor, K.P. 1997, Cosmic Winds and the Heliosphere, Jokipii, Sonnet, Giampapa (eds.), (Univ. Ariz. Press: Tucson)

Clarke, F.J., Tinney, C.G. \& Covey, K.R. 2002, MNRAS332, 361

Delfosse, X., Forveille,T., Perrier,C., \& Mayor,M., 1998, A\&A, 331, 581

Fleming, T.A., Giampapa, M.S., Schmidt, J.H.M.M., \& Bookbinder, J.A. 1993, ApJ, 410, 387

Gelino, C.R., Marley, M.S., Holtzman, J.A., Ackerman, A.S. \& Lodders, K. 2002, ApJ, 577433 
Gizis, J.E., Monet, D.G., Reid, I.N., Kirkpatrick, J. D., Liebert, J. \& Williams, Rik J. 2000, AJ, 120, 1085

Gizis, J.E., Reid, I.N. \& Hawley, S.L. 2002, AJ, 123, 3356

Hawley, S.L., Gizis, J.E. \& Reid, I.N. 1996, AJ, 112, 2799

Hawley, S.L., \& Johns-Krull, C.M. 2003, ApJ, 588, L109

James, D. J., Jardine, M. M., Jeffries, R. D., Randich, S., Collier Cameron, A., \& Ferreira, M. 2000, MNRAS, 318, 1217

Jardine, M. \& Unruh, Y.C. 1999, A\&A, 346, 883

Jayawardana, R., Mohanty, S. \& Basri, G. 2002, ApJ, 578, L141

Kraft, R.P. 1967, ApJ, 150, 551

Linsky, J.L., Wood, B.E., Brown, A., Giampapa, M.S., \& Ambruster, C. 1995, ApJ, 455, 670

Martín, E.L., Zapatero-Osorio, M.R. \& Rebolo, R. 1996, Ninth Cambridge Workshop on Cool Stars, Stellar Systems, and the Sun, A.S.P. CS-109, R. Pallavicini \& A. K. Dupree, (eds.), 615

Martín, E.L. \& Bouy, H. 2003, New Astronomy, 7, 595

Meyer, F. \& Meyer-Hofmeister, E. 1999, A\&A, 341, L23

Mohanty, S., Basri, G., Shu, F., Allard, F. \& Chabrier, G. 2002, ApJ, 571, 469

Mohanty, S. \& Basri, G. 2003, ApJ, 583, 481

Mohanty, S., Basri, G., \& Jayawardhana, R. 2004, ApJ, submitted

Montesinos, B., Thomas, J.H., Ventura, P., \& Mazzitelli, I. 2001, MNRAS, 326, 877

Mullan, D.J., \& McDonald, D. 2001, ApJ, 559, 353

Neuhauser, R., Bricenño, C., \& Comeron, F. 1999, A\&A, 343, 883

Noyes, R.W., Hartmann, L.W., Baliunas, S.L., Duncan, D.K. \& Vaughan, A.H. 1984, ApJ, 279, 763

Pizzolato, M., Maggio, A., Micela, G., Sciortino, S., \& Ventura, P. 2003, A\&A, 397,147

Reid, I.N., Kirkpatrick, J.D., Gizis, J.E. \& Liebert, J. 1999, ApJ, 527, L105

Reid, I.N., Kirkpatrick, J.D., Liebert, J., Gizis, J.E., Dahn, C.C. \& Monet, D.G. 2002, AJ, 124, 519

Rutledge, R.E., Basri, G., Martin, E.L., \& Bildsten, L. 2000, ApJ, 527, L105

Saar, S. 1996, IAU Symposium 176 (K. Strassmeier \& J. Linsky, eds.), Kluwer:Dordrecht, 237

Stauffer, J.R., Hartmann, L., Prosser, C.F. et al. 1997, ApJ, 479, 776

Terndrup, D.M., Krishnamurthi, A., Pinsonneault, M.H. \& Stauffer, J.R. 1999, AJ, 118, 1814

Tinney, C.G. \& Reid, I.N. 1998, MNRAS, 301, 1031

White, R. \& Basri, G. 2003, ApJ, 582, 1109

Zapatero-Osorio, M.R., Béjar, V.J.S., Martín, E., et al. 2002a, ApJ, 569, L99

Zapatero-Osorio, M.R., Béjar, V.J.S., Martín, E., et al. 2002b, ApJ, 578, 536 

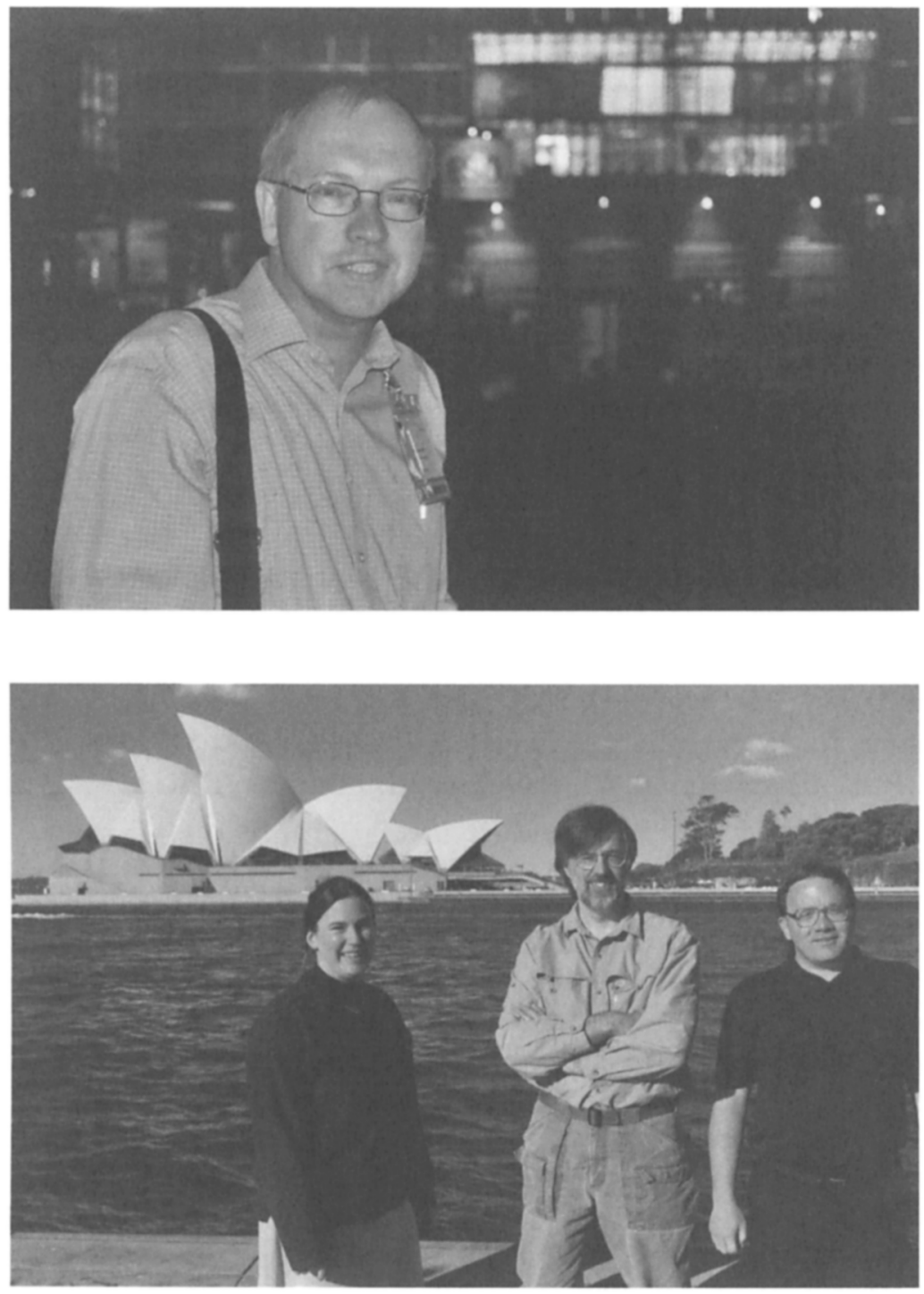